R. Brooks and R. Tse

Nagoya Math. J.

Vol. 107 (1987), 13-24

\title{
ISOSPECTRAL SURFACES OF SMALL GENUS
}

\author{
ROBERT BROOKS* AND RICHARD TSE
}

\section{Isospectral Surfaces of Small Genus}

In this note, we will construct simple examples of isospectral surfaces. In what follows, we will use the term "surface" to mean a surface endowed with a Riemannian metric, while the term "Riemann surface" will be reserved for a surface endowed with a metric of constant curvature. We will show:

TheOREM 1. There exist pairs of surfaces $S_{1}$ and $S_{2}$ of genus 3 , such that $S_{1}$ and $S_{2}$ are isospectral but not isometric.

TheOREm 2. There exist pairs of Riemann surfaces $S_{1}$ and $S_{2}$ of genus 4 and 6, which are isospectral but not isometric.

THEOREM 3. There exist unoriented surfaces $S_{1}$ and $S_{2}$ of Euler characteristic $\chi\left(S_{1}\right)=\chi\left(S_{2}\right)=-6$ which are isospectral but not isometric.

The problem of finding isospectral Riemann surfaces is an old one. The first explicit example, of genus 24, was found by Vigneras [6] through consideration of the arithmetric of quaternion algebras. Soon afterwards, Sunada [5] found a quite general approach to the subject, which we will discuss below. His method involves reducing isospectral properties to certain group-theoretic properties of finite groups. His method was taken up by Buser [3], who was able to construct isospectral Riemann surfaces of genus 5 , and all genera $\geq 7$. Buser worked with the finite group which is the semi-direct product $(Z / 8) \times(Z / 8)^{*}$.

We will show in Section 4 below how to extend Buser's work in certain directions.

To prove Theorem 1 , however, we will replace $(Z / 8) \times(Z / 8)^{*}$ with the group $S L(3, Z / 2)$. For reasons which we will explain below, $S L(3, Z / 2)$ should be close to optimal for this problem.

Received January 4, 1986.

* Partially supported by NSF grant DMS-8501300, Alfred P. Sloan fellow. 
We have made an extensive but by no means exhaustive search of finite groups likely to produce examples of isospectral surfaces of genus 2. All of the groups we studied turned out to be incapable of giving such examples. It should be possible, using the classification of finite simple groups, to decide whether or not the technique used here is capable of producing genus 2 examples, but such a question is outside the scope of this paper.

We would like to thank Robert Guralnick and David Wales for their invaluable assistance on the group theoretic aspects of this paper.

\section{$\S 1$. Finite groups and the Sunada theorem}

In [5], Sunada gives a general technique for constructing isospectral manifolds. The idea is to model this problem on an analogous problem in the theory of finite groups. To that end, let $G$ be a finite group, and $H_{1}$ and $H_{2}$ two subgroups of $G$. We will say that the triple $\left(G, H_{1}, H_{2}\right)$ satisfies the conjugacy condition if the following condition hold:

$\left(^{*}\right)$ (i ) For every conjugacy class [g], $g \in G$,

$$
\#\left([g] \cap H_{1}=\#\left([g] \cap H_{2}\right)\right.
$$

(ii) $H_{1}$ is not conjugate to $H_{2}$ in $G$.

Then Sunada shows:

TheOREm ([5]). Suppose that $\left(G, H_{1}, H_{2}\right)$ satisfies the conjugacy condition, and let $M$ be a compact manifold with a surjective homomorphism $f$ : $\pi_{1}(M) \rightarrow G$.

If $M^{1}$ and $M^{2}$ are the Riemannian coverings of $M$ with $\pi_{1}\left(M^{i}\right)=f^{-1}\left(H_{i}\right)$, then $M^{1}$ is isospectral to $M^{2}$.

Furthermore, for a generic on $M, M^{1}$ will not be isometric to $M^{2}$.

We observe that it is not important for Sunada's argument that $M$ be a smooth manifold. It suffices to assume that there is a manifold $M^{0}$ with $G$ acting on $M^{0}$ by isometries, so that $M=M^{0} / G$ in the sense of orbifolds. Similarly, we understand the homomorphism $\pi_{1}(M) \rightarrow G$ in the sense of orbifold fundamental groups. It will, however, be important that $M^{1}$ and $M^{2}$ are smooth, so that $H_{1}$ and $H_{2}$ act freely on $M^{0}$.

The problem of characterizing triples $\left(G, H_{1}, H_{2}\right)$ satisfying the conjugacy condition has been studied by a number of people, in particular [4]. In [4], the problem of which index $\left[G: H_{1}\right]=\left[G: H_{2}\right]$ can occur is 
studied, and in particular it is shown that

Theorem ([4]). Suppose $\left(G, H_{1}, H_{2}\right)$ satisfy the conjugacy condition. Then $\left[G: H_{1}\right] \geq 7$.

Furthermore, there examples are of such triples with $\left[G: H_{1}\right]=p, p a$ prime number, for all $p$ of the form $p=\left(q^{a}-1\right) /(q-1)$ with $q$ a prime and $a \geq 3$. In particular, there are examples with $\left[G: H_{1}\right]=7$.

As examples, we may take $G=\operatorname{PSL}(a, q)$ and $H_{1}$ and $H_{2}$ the following subgroups:

$$
H_{1}=\left(\begin{array}{cccc}
* & * & * & * \\
0 & & \\
0 & * & *
\end{array}\right) \quad H_{2}=\left(\begin{array}{cccc}
* & 0 & 0 & 0 \\
* & & & \\
* & * &
\end{array}\right)
$$

It is shown in [2], or can be seen by a direct elementary argument, that $\left(G, H_{1}, H_{2}\right)$ has the desired properties.

We will now specialize to the case $p=7$, so that $q=2$ and $a=3$ in the above example. We will need some elementary facts about the group $P S L(3, Z / 2)=S L(3, Z / 2)$.

Lemma 1. Let $g \in S L(3, Z / 2)$. Then either:

(i) g has 1 as an eigenvalue, in which case $g$ is of order 1,2,3 or 4 or

(ii) $g$ is of order 7 .

Proof. Consider the characteristic polynomial $c(t)$ of $g$. It is of degree 3.

It $c(t)$ is not irreducible over $Z / 2$, it has a root, which then must be 1. If $c(t)=(t-1)^{3}$, then $g$ must be of order 1,2 , or 4 . If $c(t)$ has an irreducible factor of degree 2 , then its roots are primitive cube roots of 1 , and $g$ is of order 3. Finally, if $c(t)$ is irreducible, then its roots are primitive 7 th roots of unity, and $g$ has order 7 .

LEMma 2. There are generators $A$ and $B$ of $G$ with the property that $A, B$ and $A B$ are all of order 7 .

Proof. We begin with generators

$$
X=\left(\begin{array}{lll}
1 & 1 & 0 \\
0 & 1 & 1 \\
0 & 0 & 1
\end{array}\right) \text { and } Y=\left(\begin{array}{lll}
0 & 1 & 0 \\
0 & 0 & 1 \\
1 & 0 & 0
\end{array}\right)
$$


It is well-known that $X$ and $Y$ generate $S L(3, Z)$, so they also generate $S L(3, Z / 2)$.

We observe that if $\langle X, Y\rangle$ generate a group, then so do $\langle X Y, Y\rangle$ and $\langle X, X Y\rangle$. Our strategy now is to apply one of these two moves iteratively until we arrive at a basis which satisfies the conditions of Lemma 2. Along the way, we will use Lemma 1 as a simple criterion to decide when an element has order 7.

Applying two moves of the first type, we see that $\left\langle X Y^{2}, Y\right\rangle$ generates the group, where

$$
X Y^{2}=\left(\begin{array}{lll}
1 & 0 & 1 \\
1 & 1 & 0 \\
0 & 1 & 0
\end{array}\right)
$$

has order 7 .

We now apply two moves of the second kind to arrive at the generators $\left\langle X Y^{2},\left(X Y^{2}\right)^{2} Y\right\rangle$, where

$$
\left(X Y^{2}\right)^{2} Y=\left(\begin{array}{lll}
1 & 1 & 1 \\
1 & 0 & 1 \\
0 & 0 & 1
\end{array}\right)
$$

has order 7 .

Finally, we observe that for any $k$ prime to $7,\left\langle X Y^{2},\left[\left(X Y^{2}\right)^{2} Y\right]^{k}\right\rangle$ also generates the group, and $\left[\left(X Y^{2}\right)^{2} Y\right]^{k}$ will again have order 7 . We now seek $k$ so that $\left(X Y^{2}\right)\left[\left(X Y^{2}\right)^{2} Y\right]^{k}$ has order 7 , and we verify that this is the case for $k=2$. The basis

$$
A=\left(\begin{array}{lll}
1 & 0 & 1 \\
1 & 1 & 0 \\
0 & 1 & 0
\end{array}\right) \quad B=\left(\begin{array}{lll}
0 & 0 & 1 \\
1 & 0 & 0 \\
1 & 1 & 0
\end{array}\right) \quad A B=\left(\begin{array}{lll}
1 & 1 & 1 \\
1 & 0 & 1 \\
1 & 0 & 0
\end{array}\right)
$$

then establishes Lemma 2.

\section{$\S 2$. The examples}

We begin this section by considering the group $\Gamma_{0}=\Gamma_{0}(p, q, r)$ of isometries of the hyperbolic plane generated by reflections in the sides of a hyperbolic triangle with angles $(\pi / p),(\pi / q)$, and $(\pi / r)$ respectively. Let $\Gamma$ denote the subgroup of index 2 of $\Gamma_{0}$ consisting of orientation preserving isometries.

When $p, q$, and $r$ are integers, one sees from the Poincaré polygon theorem ([1]) that $\Gamma$ is a discrete group, with a fundamental domain $R$ the rectangle obtained from the triangle by reflecting it in one of its 
sides. $\Gamma$ is presented by generators and relations by

$$
\Gamma=\left\{A, B: A^{p}=B^{q}=(A B)^{r}=1\right\}
$$

where $A$ and $B$ are as shown in Figure 1.

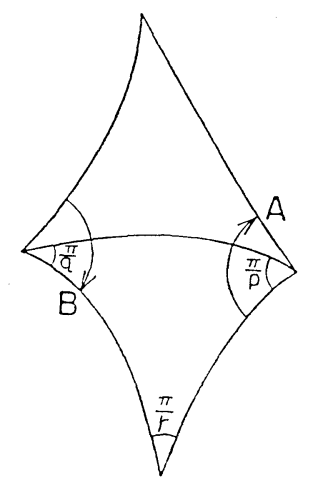

Fig. 1

We may identify $\boldsymbol{H}^{2} / \Gamma$ in the orbifold sense with the sphere with three singular points, of order $p, q$, and $r$ respectively.

We now seek a homomorphism $f: \Gamma \rightarrow G P S L(3, Z / 2)$ with the property that, if $\Gamma^{i}=f^{-1}\left(H_{i}\right)$ for $i=1,2$, we should have $H^{2} / \Gamma^{i}$ a smooth manifold. To understand this, let us fix $i=1$. Then $G / H_{1}$ may be identified with $3 \times 3$ non-zero column vectors, under the identification $g \rightarrow g\left(\begin{array}{l}1 \\ 0 \\ 0\end{array}\right)$. We may then identify $\boldsymbol{H}^{2} / \Gamma^{i}$ with a hyperbolic surface built up of seven copies of $R$ according to the action of $A$ and $B$ on the column vectors. It is easily seen that $H^{2} / \Gamma_{i}$ cannot be smooth if $A, B$ or $A B$ has an eigenvector, for then in some copy of $R$ some is glued to an adjacent side, and the angle about the fixed vertex is not $2 \pi$.

Applying Lemma 1, we see that $A, B$, and $A B \in I$ must be sent to elements of $G$ of order 7. This tells us that $p, q$, and $r$ must be of order 7. Lemma 2 then tells us how to find appropriate elements of $G$ for the images of $A, B$, and $A B$. Since the only relations in $\Gamma$ are as above, this gives us a well-defined homomorphism $f: \Gamma \rightarrow G$ having the property that $\boldsymbol{H}^{2} / \Gamma_{1}$ is smooth.

We now apply the same considerations to $H_{2}$. We may identify the coset space $G / H_{2}$ with row vectors, under the identification $g \rightarrow(1,0,0) g^{-3}$. One concludes as above that $\boldsymbol{H}^{2} / \Gamma_{2}$ is smooth. 
Fundamental polygons for $\boldsymbol{H}^{2} / \Gamma_{1}$ and $\boldsymbol{H}^{2} / \Gamma_{2}$ to which one may apply the Poincaré polygon theorem and check smoothness are shown in Figure 2.

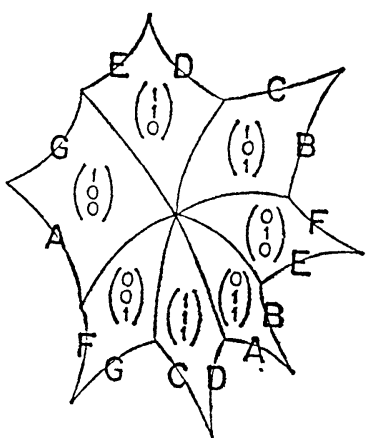

(a)

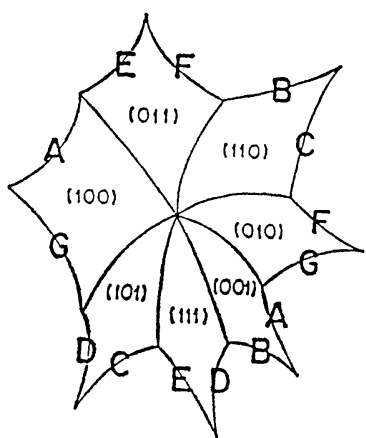

(b)

Fig. 2

Sudana's theorem now applies to tell us that $\boldsymbol{H}^{2} / \Gamma_{1}$ is isospectral to $\boldsymbol{H}^{2} / \Gamma_{2}$, and that furthermore this remains true for any choice of metric on $\boldsymbol{H}^{2} / \Gamma$.

We now calculate the genus of $H^{2} / \Gamma_{1}$. But the area of $R$ is $2 \pi-3$. $((2 \pi / 7))=(8 \pi / 7)$, and $\boldsymbol{H}^{2} / \Gamma_{1}$ has 7 copies of $R$, so $\boldsymbol{H}^{2} / \Gamma$ has area $8 \pi$. The Gauss-Bonnet theorem then tells us that $R$ has genus $g=(1 / 4 \pi)(8 \pi)+$ $1=3$.

It remains to check whether $\boldsymbol{H}^{2} / \Gamma_{1}$ is isometric to $\boldsymbol{H}^{2} / \Gamma_{2}$ when $\boldsymbol{H}^{2} / \Gamma$ is given a metric of constant curvature -1 . Since $H^{2} / \Gamma$ is a sphere with three singular points, this metric is unique.

One may see by investigating the gluing diagrams Figures 2(a) and 2(b), that they differ by a reflection. Therefore, if $\boldsymbol{H}^{2} / \Gamma$ is given a metric which is preserved by this reflection, $\boldsymbol{H}^{2} / \Gamma_{1}$ and $\boldsymbol{H}^{2} / \Gamma_{2}$ will differ by an orientation reversing isometry. Since the unique metric on $H^{2} / \Gamma$ of constant curvature -1 has this property, the metrics constructed above cannot be taken to be of constant curvature.

We now extend our examples in the following way: instead of $R$, we begin with a hexagon with gluing pattern as show in Figure 3. This gives us a group $\Gamma$ with $H^{2} / \Gamma$ a sphere with four singularities. $\Gamma$ is then given by generators and relations as

$$
\Gamma=\left\{A, B, C: A^{7}=B^{7}=C^{7}=(A B C)^{7}=1\right\} .
$$

Once again, we may apply Lemma 2 to find a homomorphism $\Gamma \rightarrow G$ 


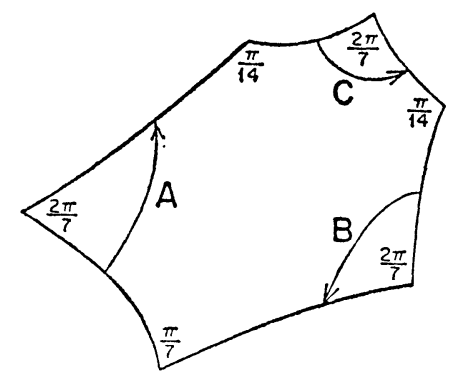

Fig. 3

in the following way: We may send $A$ and $B$ to the generators of $G$ given by Lemma 2. Then $A B$ is of order 7 in $G$, and all elements of order 7 in $G$ are conjugate. There is then an element $D$ in $G$ of order 7 such that $(A B) D$ is order 7 , and we may send $C$ to $D$. The smoothness of $\boldsymbol{H}^{2} / \Gamma_{1}$ and $\boldsymbol{H}^{2} / \Gamma_{2}$ follow exactly as above.

The area of $R$ is now $4 \pi-((8 \pi / 7))=(20 \pi / 9)$, so the genus of $\boldsymbol{H}^{2} / \Gamma_{1}$ is $(1 / 4 \pi)((7 \cdot(20 \pi / 7)))+1=6$.

We now have a one complex parameter family of hyperbolic structures for $\boldsymbol{H}^{2} / \Gamma$, and it is easily seen from an argument of [5] that for generic choice of the hyperbolic structure, $\boldsymbol{H}^{2} / \Gamma_{1}$ is not isometric to $\boldsymbol{H}^{2} / \Gamma_{2}$.

\section{§3. Isospectral surfaces of genus 4}

In this section, we modify the techniques of the previous section to construct isospectral surfaces of genus 4 .

The base surface for this construction will be a torus $M$ with one singular point. Its fundamental group is given by

$$
\pi_{1}(M)=\left\{X, Y, C: C=[X, Y], C^{k}=1\right\} .
$$

As before, we will take the group to be $S L(3, \boldsymbol{Z} / 2)$. We seek a surjective homomorphism $\pi_{1}(M) \rightarrow G$ such that $M_{1}$ and $M_{2}$ are smooth. This requirement then forces $C$ to not have 1 as an eigenvalue, so by Lemma 1, $C$ must have order 7, and hence $k=7$. We therefore seek matrices $A$ and $B$ in $\operatorname{PSL}(3, \boldsymbol{Z} / 2)$ which generate $\operatorname{PSL}(3, \boldsymbol{Z} / 2)$, whose commutator is of order 7 .

The following is easily proved:

Lemma 3. Let 
Then

$$
A=\left(\begin{array}{lll}
0 & 1 & 1 \\
0 & 1 & 0 \\
1 & 0 & 0
\end{array}\right) \quad B=\left(\begin{array}{lll}
1 & 0 & 0 \\
0 & 0 & 1 \\
0 & 1 & 1
\end{array}\right)
$$

(i) $[A, B]$ is or order 7 .

(ii) $A$ and $B$ generate $P S L(3, Z / 2)$.

Proof. (i) is verified by a direct computation. Note that $C=[A, B]$ is $\left(\begin{array}{lll}0 & 1 & 1 \\ 1 & 1 & 1 \\ 0 & 1 & 0\end{array}\right)$. To show (ii), we first observe the isomorphism $\operatorname{PSL}(3, Z / 2)=$ $P S L(2, Z / 7)$. We then observe that $A$ is of order 4 and $B$ is of order 3 .

We now claim that, for any $X, Y \in P S L(2, Z / 7)$, if $X$ is of order 4 and $Y$ is of order 7 , then $X$ and $Y$ generate $P S L(2, Z / 7)$. To see this, first note that $Y$ is a parabolic element of $P S L(2, Z / 7)$, and so fixes a unique point $p$ of the projective line $(Z / 7 \times Z / 7) /(Z / 7)^{*}$. Then $X Y X^{-1}$ fixes the point $X(p)$. If $X$ does not fix $p$, then conjugating by a linear fractional transformation sends $p$ to $(1,0)$ and $X(p)$ to $(0,1)$, so that $Y$ is conjugate to $\left(\begin{array}{ll}1 & * \\ 0 & 1\end{array}\right)$ and $X Y X^{-1}$ to $\left(\begin{array}{ll}1 & 0 \\ * & 1\end{array}\right)$. It is then clear that $Y$ and $X Y X^{-1}$ generate $P S L(2, Z / 7)$. But an element of order 4 fixes no elements in $(Z / 7 \times$ $Z / 7) /(Z / 7)^{*}$, so $X$ cannot fix $p$. We may take $X=A$ and $Y=[A, B]$ to establish (ii).

We may now calculate the hyperbolic area of $M$ to be $(2 \pi-(2 \pi / 7))$ $=(12 \pi / 7)$, so that $M_{1}$ and $M_{2}$ have area $12 \pi$, and so are of genus 4 , as desired.

In Figure 4, we have provided gluing diagrams for the surfaces $M_{1}$ and $M_{2}$. It is easily seen from these diagrams that $M_{1}$ and $M_{2}$ are not isometric for generic choices of constant-curvature metrics on $M$. Indeed.

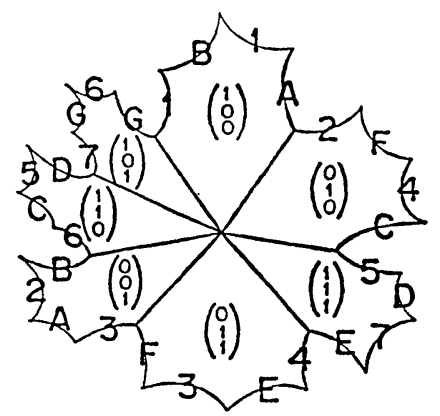

(a)

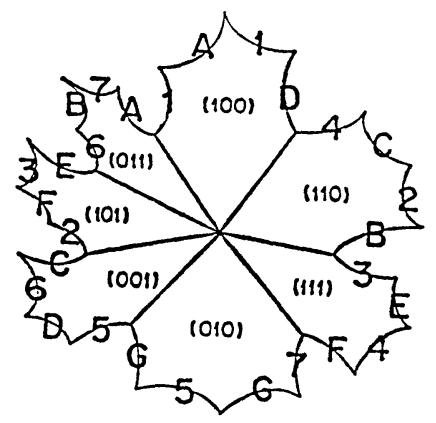

(b)

Fig. 4 
$M_{1}$ and $M_{2}$ have a number of distinguished geodesics on them. Since $B$ is of order 3 , it has a unique eigenvector. The geodesic joining the pairs of sides labeled 1 on both gluing diagrams must therefore correspond under a isometry between $M_{1}$ and $M_{2}$, for a generic metric of constant curvature on $M$. Similarly, $A$ has a unique eigenvector and a unique pair of vectors $v_{1}$ and $v_{2}$ such that $A\left(v_{1}\right)=v_{2}, A\left(v_{2}\right)=v_{1}$. It follows that for a generic metric on $M_{1}$ the geodesic joining the two pairs of sides labeled $A$ and $B$ in Figure 4(a) must correspond to the geodesic joining the pairs of sides labeled $E$ and $F$ in Figure 4(b). But the first of these crosses the geodesic joining the sides labeled 1, while the second does not meet the geodesic joining the sides labeled 1 .

\section{§4. Further examples}

In this section, we return to the group $G=(Z / 8) \times(Z / 8)^{*}$, which was considered by Sunada [5] and Buser [4]. We will show:

THEOREM 3. There exist closed, unoriented manifolds $M_{1}$ and $M_{2}$ which are isospectral and $\chi\left(M_{1}\right)=\chi\left(M_{2}\right)=-6$.

Our method will be completely analogous to the method of Section 1 and Section 2 above.

Let us begin with a study of the group $G$. For convenience, we will write elements of $G$ in the form $(a, b), a \in(Z / 8)^{*}=\{1,3,5,7\}$ and $b \in Z / 8$. The composition rule is given by

$$
(a, b) \cdot\left(a^{\prime}, b^{\prime}\right)=\left(a a^{\prime}, b \cdot a^{\prime} b^{\prime}\right) .
$$

Let $H_{1}$ be the subgroup $\{(1,0),(3,0),(5,0),(7,0)\}$ and $H_{2}$ the subgroup $\{(1,0),(3,4),(5,5),(7,0)\}$. It is easy to verify that $\left[G: H_{1}\right]=8$, and that $\left(G, H_{1}, H_{2}\right)$ satisfies the conjugacy condition.

We may identify the coset spaces $G / H_{1}$ and $G / H_{2}$ with the integers (mod 8) in the following way: given $(a, b) \in G$, then there is a unique $c=$ 0 or 4 such that $(a, c) \in H_{i}$. Then $(a, b) \cdot(a, c)=(1, a b+c)$, and we map $(a, b)$ to $a b+c(\bmod 8)$.

We need some elementary results on the group structure of $G$ analogous to Lemma 1:

Lemma 3. Let $(a, b) \in G$. Then exactly one of the following holds:

(i) $(a, b)$ is of order 1 , in which case $a=1, b=0$. 
(ii) $(a, b)$ is of order 2, in which case
(a) $a=1, b=4$
(b) $a=3, b=0,2,4$, or 6
(c) $a=5, b=0$ or 4
(d) $a=7, b$ is arbitrary

(iii) $(a, b)$ is of order 4 , in which case
(a) $a=1, b=2$ or 6
(b) $a=3, b=1,3,5$ or 7
(c) $a=5, b=2$ or 6

(iv) $(a, b)$ is of order 8 , in which case
(a) $a=1, b=1,3,5$ or 7
(b) $a=5, b=1,3,5$ or 7 .

We now need

LEMma 4. $g \in G$ acts freely on the cosets of $G / H_{i}$ if and only if

(a) $g$ is of order 2, in which case

$$
a=1 \text { and } b=4 \text { or } a=7 \text { and } b=1,3,5 \text {, or } 7
$$

or

(b) $g$ is of order 4 or 8 .

Froof. Suppose first that $i=1$. We then consider the equation

$$
(a, b)(1, c)(a, 0)=(1, c) .
$$

This is the same as $a(b+c)=c$, or

$$
a b \equiv(1-a) c \quad(\bmod 8) .
$$

Since $1-a$ is even, this will be solvable if and only if the power of 2 dividing $a-1$ also divides 5. This establishes 4(a). To show 4(b), we observe that if $(a, b)$ is not of order 2 , then $(a, b)^{2}=(1, c)$ for $c \neq 0$. Since $(1, c)$ acts freely, $(a, b)$ will act freely if and only if $(a, b)$ does not fix a coset, and apply the above considerations to prove $4(b)$. For $i=2$, the consideration are the same.

We now consider the polygon $R$ showed in Figure 5, with the gluing patterns shown. $C$ denotes an order 2 reflection.

If $M$ denotes the orbifold so obtained, its orbifold fundamental group is given by

$$
\left\{A, B C: C^{2}=A^{p}=B^{q}=\mathrm{id}, C(A B) C^{-1}=A B\right\}
$$




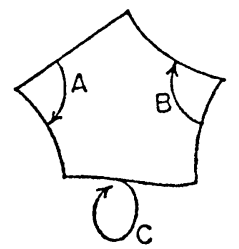

Fig. 5

To obtain manifolds for $M_{1}$ and $M_{2}$, we must have that $C, A$, and $B$ act freely on the cosets of $G / H_{i}$. In particular, $C=(1,4)$ or (7, odd). If $C=(1,4)$, then $A$ and $B$ cannot be chosen so that $A, B$, and $C$ generate $G$. So, up to conjugation, we may assume that $C=(7,1)$.

The equation $C(A B)+C^{-1}=A B$ now forces $A B$ to be of the form $(a, b)$, where $2 b=1-a$. We may choose $A=(5,5)$ and $B=(1,1)$.

With this choice, one can now verify directly that $M_{1}$ and $M_{2}$ are both non-oriented manifolds with $\chi\left(M_{i}\right)=-6$, as desired. We may construct gluing diagrams as in Figure 6.
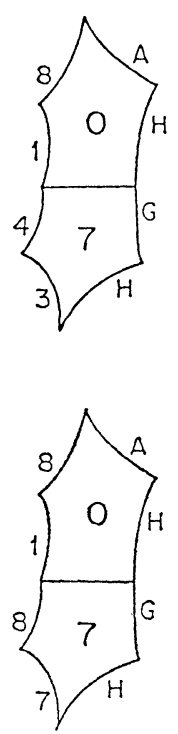

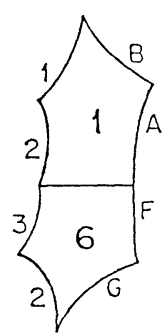

(a)

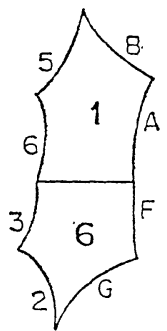

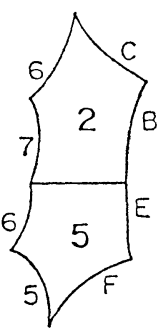
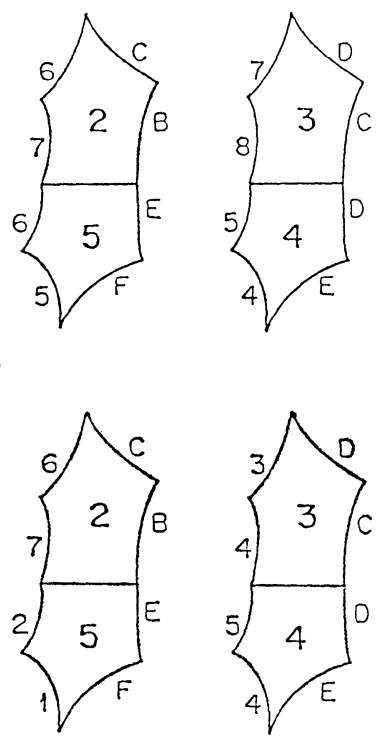

(b)

Fig. 6

Since there is no canonical metric on an unoriented surface, we are free to choose an arbitrary metric on $M$, completing the proof of the theorem. 


\section{REFERENCES}

[1] A. Beardon, The Geometry of Discontinuous Groups, Springer Verlag 1983.

[2] R. Brooks, On manifolds of negative curvature with isospectral potentials, Topology, 26 (1987), 63-66.

[ 3 ] P. Buser, Isospectral Riemann surfaces, Ann. Inst. Fourier, XXXVI (1986), 167192.

[ 4 ] R. Guralnick, Subgroups inducing the same permutation representation, J. Algebra, 81 (1983), 312-319.

[5] T. Sunada, Riemannian coverings and isospectral manifolds, Ann. of Math., 121 (1985), 169-186.

[6] M. F. Vigneras, Variétés riemannienes isospectrales et non isometriques, Ann. of Math., 112 (1980), 21-32.

Department of Mathematics

University of Southern California

Los Angeles, California 90089-1113

U.S.A. 